\title{
Competências de Estudo dos Estudantes Universitários: Estudo comparativo entre uma universidade portuguesa e brasileira
}

Competencias de los estudiantes universitarios: estudio comparativo entre una universidad portuguesa y brasileña

Rubia Salheb Fonseca, Joaquim Escola, Armando Loureiro, Amâncio Carvalho'

\section{Resumo}

Para se compreender as competências de estudo e autorregulação no ensino superior, há que se colocar o estudante como centro do seu processo de aprendizagem. Neste estudo analisou-se a relação entre o perfil de competências de estudos dos estudantes de universidades de dois países (Brasil e Portugal). Trata-se de um estudo comparativo, transversal, de abordagem quantitativa, com 1240 estudantes universitários, aos quais foi aplicado um inquérito por questionário. O tratamento de dados foi efetuado com recurso ao SPSS (23.0).

\section{Palavras-chave}

Estudantes Universitários, universidade, competência em Estudo, processo de Ensino-Aprendizagem.

\section{Resumen}

Para comprender las competencias de estudio y autorregulación en educación superior, el estudiante debe situarse en el centro de su proceso de aprendizaje. Este estudio analizó la relación entre el perfil de competencias de estudios de los estudiantes universitarios en dos países (Brasil y Portugal). Se trata de un estudio comparativo, transversal, cuantitativo, con 1240 estudiantes universitarios, sobre los que se realizó un sondeo por encuesta. El tratamiento de los datos fue realizado mediante el programa SPSS (23.0).

\section{Palabras clave}

Estudiantes universitarios, universidad, competencias de estudio, proceso de enseñanza-aprendizaje.

\section{Abstract}

In order to understand the competences of study and self-regulation in higher education, the student must be placed at the center of his learning process. This study analyzed the relationship between the proficiency profile of studies of students from universities in two countries (Brazil and Portugal). This is a cross-sectional, cross-sectional, quantitative study involving 1240 college students, to whom a questionnaire survey was applied. Data processing was done using SPSS (23.0).

Key words

University Students, university, study skills, teaching-learning process.

1 Universidade de Trás-os-Montes e Alto Douro (UTAD), Portugal, Rubia Salheb Fonseca, rubiasalf@yahoo.com.br; Joaquim Escola, jescola@utad.pt; Armando Loureiro, aloureiro@utad.pt; Amâncio Carvalho, amancioc@utad.pt 


\section{Introdução}

A obtenção do diploma de curso superior é uma meta para grande parte dos jovens como se pode notar no crescimento das taxas de inscrição no Ensino Superior (ES) em Portugal. De acordo com a Pordata (2017) o crescimento do número de inscritos permitiu a gradual qualificação da população portuguesa, tendo a percentagem de população residente com um grau superior aumentado de 0.5\% em 1960 (49.065 indivíduos) para 12\% em 2011 (1.244.742 indivíduos).

Ainda fazendo menção à Pordata (2014), a população residente em Portugal no ano de 2014 registrou o número de 10.401.1, tendo como população residente de 15 e mais anos com ensino superior $16.5 \%$ e, em 2015, 17.1\%. O número de alunos diplomados era de 72.906 e o total dos que efetuaram a matrícula pela primeira vez no ES foi de 104.255. Destes, 46.120 eram homens e 57.518 mulheres, demonstrando mais uma vez a permanência deste quadro de ascensão das mulheres na história dos alunos no ES em Portugal.

No Brasil em 2004, a parcela de jovens de 18 a 24 anos no Ensino Superior era de 32,9\% e cresceu para 58,5\% em 2014 de acordo com o Instituto Brasileiro de Geografia e estatística- IBGE (2015) na Síntese de Indicadores Sociais (SIS) de 2015. Segundo dados do IBGE (2015), no Brasil, o sistema de Educação Superior ampliou-se expressivamente nos últimos anos, gerando mudanças significativas não somente na dinâmica de funcionamento das instituições de ensino superior (IES) mas também no perfil dos estudantes que nelas ingressam (Almeida, Marinho-Araujo, Amaral \& Dias, 2012). Em termos de quantidade de estudantes, a Educação Superior brasileira era a maior da América Latina e a quinta maior do mundo em 2010, com 6,7 milhões de matrículas (UNESCO, 2011). O sistema chegou a registrar um total de 2.391 instituições (87,4\% privadas e 12,6\% públicas) e 7.305.977 alunos de graduação matriculados em cursos presenciais e a distância no ano de 2013. No atual cenário da Educação Superior brasileira, no censo de 2014, quanto ao gênero, a maior participação é feminina, que corresponde a 54,7\% dos ingressantes, 55,5\% dos estudantes matriculados e 59\% dos concluintes (INEP, 2014). A idade modal dos estudantes matriculados é de 21 anos nos cursos de graduação presenciais e 32 anos nos cursos a distância. No Brasil em média há 2,5 alunos matriculados na rede privada para cada aluno matriculado na rede pública em cursos presenciais. Mais de 3,1 milhões de alunos ingressaram em cursos de educação superior de graduação. Desse total 82,4\% em instituições privadas. O País tem projetos que facilitam o acesso de alunos e professores à educação superior, ajudando a melhorar a qualidade do ensino.

A partir desse quadro, encontra-se uma diversidade de contextos que, por sua vez, permitem aos estudantes diferentes experiências. Ingressar na universidade acarreta grandes e novos desafios afetivos, cognitivos e sociais. Os anos que os estudantes passam na universidade são importantes, tanto para o desenvolvimento pessoal, quanto para a formação profissional, refletindo no desenvolvimento da própria sociedade onde irão atuar quando graduados. Dada essa diversidade, é importante conhecer qual é o perfil universitário do século xxI. Como estabelece a UNESCO (2011), «Os alunos não são o objeto da educação, mas sujeitos com direito a uma educação que potencie ao máximo o seu desenvolvimento como pessoas, e lhes permita inserir-se e influir na sociedade em que estão imersos» (p. 10). As exigências de qualificação profissional e de aprendizagem contínua (Jenschke, 2003; Soares, 2000), somadas à expansão e à democratização do acesso ao Ensino Superior (Ministério da Educação, 2007) têm estimulado o ingresso de um número cada vez maior de estudantes nas universidades. Como consequência disso, constata-se a heterogeneidade dos estudantes universitários em termos de idade, classe social, procedência geográfica e competências de estudo (Macedo, Trevisan, Trevisan \& Macedo 2005; Soares, 2002; Zago, 2006). Ao mesmo tempo que se observa a ampliação do sistema de educação superior, verifica-se 
a necessidade de apoio e orientação aos universitários, no decorrer de seus anos de formação, a fim de facilitar sua aprendizagem, sucesso escolar e desenvolvimento psicossocial (Almeida \& Soares, 2004). Nesse sentido, surge o interesse em conhecer em maior profundidade as competências de estudo, dos alunos (Ferreira, Almeida, \& Soares, 2001).

Os estudos de Nardelli et al., (2013) afirmam que poucas vezes, durante a formulação do processo ensino-aprendizagem, dados relativos aos alunos têm sido considerados relevantes, porém a criação de um perfil do ingressante pode ajudar a universidade no estabelecimento de políticas que interfiram positivamente na vida do estudante, bem como proporcionar subsídios para uma melhor interação entre professor-aluno e para o processo de ensino-aprendizagem. Os estudos de Sarriera et al., (2012) também apontaram para a importância da identificação das características, pessoais e institucionais, para a melhor integração dos alunos nos seus contextos universitários. Para o perfil de competências de estudo, Silva e Silva (2015) defendem que a criação de estratégias institucionais que favoreçam os hábitos de estudo dos estudantes é fundamental para melhorar o desempenho acadêmico no ES. Segundo Méndez (1999), os métodos de estudo são considerados como um utensílio vantajoso para aqueles estudantes que o utilizam, o qual, os pode ajudar a aperfeiçoar os seus resultados escolares, ao mesmo tempo que podem aprender e criar objetivos, a planificar as suas atividades e distribuir seu tempo. No mesmo seguimento, Competência de estudo, designado na literatura como Study Skills, tem sido objeto de específica atenção por parte de pesquisadores na área da Educação e na Psicologia Educacional (Almeida, 2002; Credé \& Kuncel, 2008). Essas competências (de estudo) são definidas por Credé e Kuncel (2008) como a capacidade de apropriação das estratégias de estudo e método que o aluno tem, capacidade de gestão de tempo e de outros recursos, visando responder questões acadêmicas.

Para se compreender as competências de estudo no ensino superior, há que se colocar o estudante como centro do seu processo de aprendizagem e como ator principal. É nesse sentido que Zimmerman (2000; 2008) e Zimmerman e Martinez-Pons (1988), precursores da teoria da autorregulação, colocam como centrais os processos de autorregulação da aprendizagem, definindo-os como autodirecionados e de crenças pessoais que possibilitam ao estudante transformar competências cognitivas em competências de desempenho. Veiga Simão (2004) define a autorregulação da aprendizagem como o processo em que o aluno estabelece metas que se misturam com suas expectativas, desenvolve estratégias para alcançá-las, criando condições para que se efetive a aprendizagem. As estratégias vinculadas à autorregulação são planejamento, monitoramento e autoavaliação. (Zimmerman, 2000)

O planejamento das atividades refere-se à definição de objetivos e metas, alguns exemplos de estratégias de planejamento são: passar os olhos rapidamente no texto antes de ler, formular questões antes de ler um texto, fazer uma análise prévia das informações (Pintrich, 1999, 2004).

As estratégias de monitoramento alertam o estudante quanto a possíveis problemas relativos à sua atenção ou compreensão que podem ser reparados com alguma estratégia de regulação. Dentre as atividades de controle, pode-se destacar o monitoramento de atenção ao ler um texto por meio de questionamentos sobre o material para verificar a compreensão, além do monitoramento de velocidade de leitura a fim de ajustar o tempo disponível, de controlar os comportamentos, o ambiente físico e seus próprios processos internos. (Pintrich, 1999, 2004; Pintrich \& et al., 1993).

A autoavaliação consiste «no juízo de valor que o aluno faz do rendimento educativo que obteve» (Carrasco,1989:22). Segundo Ferreira (2007:111), é «através da reflexão que o aluno modifica a sua ma- 
neira de atuar, atribuindo um sentido ao seu processo de aprendizagem». Portanto, a regulação das atividades ou autoavaliação, ocorre após o desempenho, refere a um ajuste contínuo das ações cognitivas que se realizam em função de um controle prévio (Rojas, 2008; Schreiber, 2005).

Os estudantes do ensino superior desconhecem muitas vezes as regras de funcionamento das instituições que escolheram, desconhecem os cursos e o seu planeamento curricular, enfrentando novas disciplinas e uma diversidade de métodos de avaliação (Pinheiro, 2003). Na atual Sociedade da Informação e do Conhecimento é importante aceder, analisar e utilizar a informação de forma adequada. Para isso, segundo as diretrizes do Espaço Europeu de Educação Superior (EEES), é necessária uma série de competências e habilidades relacionadas com a pesquisa, avaliação, gestão, uso e difusão da informação (Lopes \& Pinto, 2011; Pinto, 2010, 2011, 2012). As dificuldades dos estudantes universitários no uso e gestão de informação é uma realidade presente nos nossos dias. Acumulam muitos dados, mas não sabem como estruturar e interrelacionar o conhecimento. De acordo com Read (1999), para que um aluno da universidade seja bem-sucedido na aprendizagem académica, deve ser capaz de interpretar e compreender a informação escrita, de ter raciocínios abstratos, de refletir e de generalizar conceitos, de colocar questões e esclarecer dúvidas bem como expor conhecimentos oralmente, por escrito e de forma numérica (cit. in Santos, 2000). Cabe destaque que, se entende por competência de estudo como a capacidade do estudante em se apropriar de alguns recursos como, por exemplo, estratégias e métodos de estudo e capacidade de gestão de tempo, com o objetivo de realizar as tarefas acadêmicas (Credé \& Kuncel, 2008). Robbins et al., (2004) acrescentam que as competências de estudo são comportamentos que estão diretamente relacionados com um desempenho produtivo e determinante do sucesso acadêmico.

No dizer de Ferreira et al., (2001:8) «a educação universitária deve promover o desenvolvimento de competências académicas, cognitivas e pessoais. Estas competências devem ser promovidas através de atividades curriculares e extracurriculares, tendo em vista a preparação dos alunos para a vida ativa, considerando as coordenadas histórico-socio-culturais e geográficas em que vivem». Ainda segundo Ferreira et al., (2001: 8) a Universidade deve encarar o «sucesso académico dos seus estudantes para além dos resultados obtidos em cada disciplina, devendo tomar isso em consideração desde o $1^{\circ}$ ano dos seus cursos». Ao reduzir o sucesso académico dos estudantes às suas classificações curriculares torna-se, muitas vezes, o desenvolver competências nos alunos em apenas reproduzir informação, o que conduz a enfatizar pouco a preparação dos estudantes para que possam, no futuro, se integrarem em ambientes profissionais e sociais. Zabalza (2004) destaca como função do Ensino Superior a qualificação de pessoas, integrando na formação superior as dimensões que o estudante pode desenvolver e regular como consequência das próprias experiências e aponta os seguintes caminhos formativos: a) aprender a aprender, b) aprender a fazer, c) aprender a ser, d) aprender a (con)viver. Na atualidade, a universidade é considerada uma Multipurpose University, de acordo com os documentos da OCDE (1981, cit. in Oliveira, 2006), permitindo não só a reprodução de conhecimento, mas igualmente a criação, a difusão e a inovação.

Segundo Maestro (2014), os acadêmicos deste século, nativos da geração Y (nascidos entre 1980 a 2000), têm mais facilidade em lidar com tecnologias, ditam as regras, têm ideias inusitadas, inovam, porém nada os satisfaz, são imediatistas e de pouca paciência. Nesse contexto estão os universitários de hoje: criativos e em constante busca pelo conhecimento, também característica do mercado de trabalho atual. Nunca se produziu tanto conhecimento como nesta era, e somente estarão no mercado aqueles que detêm de conhecimento, Maestro (2014) ressalta ainda as mudanças no mercado de trabalho, apontando a 
forte ascensão na prestação de serviço e não mais na agricultura e nas fábricas, a exemplo do início do século passado, mostrando que no mundo pós-industrial o trabalho não é mais como obrigação opressora, mas, sobretudo como um prazer criativo e estimulante. Educar é um ato complexo, onde não se pode menosprezar o fato de que o aluno é o objeto de estudo, este também com todo o seu contexto, sua vivência e complexidade e diante de tais, a educação deve levar em consideração as dimensões deste ser em sala de aula. É no âmbito desta problemática que este estudo tem como objetivo geral analisar a relação entre o perfil de competências de estudos, dos estudantes universitários das duas universidades dos dois países (Brasil e Portugal), participantes no estudo.

\section{Aspectos metodológicos}

Desenvolveu-se um estudo comparativo, transversal, de abordagem quantitativa (Fortin et al., 2013). A amostra incluiu 1240 alunos que frequentavam 10 cursos (Biologia, Ciências do Desporto, Comunicação e Multimédia, Educação Básica, Enfermagem, Engenharia Civil, Engenharia Eletrotécnica e de computadores, Engenharia Informática, Línguas, Literatura e Cultura e Psicologia) de uma Universidade de Portugal e uma Brasileira, que estavam presentes no momento de recolha de dados e aceitaram participar no estudo. Estabelecemos como critérios de seleção, ser aluno dos cursos selecionados e frequentar o $1^{\circ}$, $2^{\circ}$ ou $3^{\circ}$ ano dos cursos.

Para a recolha de dados foi aplicado um questionário de autopreenchimento, com questões abertas e fechadas, de escolha múltipla, com questões abertas construídas e validadas para o efeito, através de um teste piloto. A primeira parte inclui um conjunto de questões de caracterização sociodemográfica e a escala de Graffar (1956), e a última, integra a escala de competências de estudo de Almeida \& Joly (2013) e questões acerca do estudo autónomo. A escala de competências de estudo pretende avaliar os métodos de estudo e as abordagens à aprendizagem dos estudantes no ensino superior. É composta por 16 itens do tipo Likert com seis pontos, de discordo totalmente (1 ponto) até concordo totalmente (6 pontos), agrupando-se em 3 fatores.

O fator 1, designado Comportamentos Estratégicos de Planejamento contém cinco itens que se referem a decisões prévias relativas à forma como o estudante organiza o seu estudo, integrando os itens 3, 7, 8, 9 e 14. O fator 2, designado Comportamentos Estratégicos de Monitoração, inclui cinco itens, objetiva realizar a automonitoração pela auto-observação do desempenho durante a execução de atividades de estudo é composto pelos itens 4, 6, 11, 15 e 16. Por último, o fator 3, designado Comportamentos Estratégicos de Auto-avaliação contém seis itens, reportando-se a comportamentos e a preocupações dos alunos no sentido de viabilizarem um estudo competente e bem sucedido, sendo por isso dependentes da existência de uma autoreflexão por parte do estudante acerca de seu desempenho, quer seja antes, durante ou depois de estudar, sendo composto pelos itens 1, 2, 5, 10, 12 e 13 .

No dizer de Almeida \& Joly (2013) a escala revela boas qualidades pesicométricas. A análise fatorial exploratória revelou que os itens se agruparam nos três fatores revelando cargas fatoriais bastante altas que variaram de 0,49 a 0,79 e comunalidade acima de $35 \%$. O fator 1 explica 17,7 \% da variância, o fator 2 explica $17,68 \%$ de variância e o fator 3 explica $17,9 \%$ da variância. A análise da precisão foi realizada por meio da consistência interna dos itens (alfa de Cronbach) para cada um dos três fatores. Constataram-se bons índices de precisão, sendo de 0,75 para o fator 1, 0,77 para o fator 2 e em 0,76 para o fator 3. Antes da aplicação do questionário foi realizado um pedido de autorização para a realização do estudo à comissão de ética das duas universidades, que nos deram o seu parecer favorável (n $\left.n^{\circ} 7 / 2016\right)$ Portugal e plata- 
forma Brasil ( $\mathrm{n}^{\circ}$ 1.901.179). De seguida foi agendada por email a recolha de dados com os professores de cada um dos cursos. A investigadora deslocou-se às salas de aula, tendo informado os estudantes sobre o objetivo do estudo, e realizada a entrega e recolha dos questionários em sala de aula. Para o tratamento dos dados utilizou-se o software estatístico Statistical Package for the Social Sciences (SPSS), versão 22.0 para Windows, no qual foi construída uma base de dados e os mesmos foram editados. Recorremos à estatística descritiva, utilizando as frequências absolutas e relativas, a média e o desvio-padrão da idade e à estatística inferencial com os testes não paramétricos de $\mathrm{x}^{2}$, Mann-Whitney e Kruskal-Wallis. O nível de significância adotado foi de 5\% (Marôco, 2014).

\section{Resultados e Discussão}

O perfil sociodemográfico abrangeu as variáveis sexo, grupo etário, estado civil, coabitação em tempo de aulas, tipo de habitação, meio de deslocação para a universidade, manutenção financeira, nível socioeconómico (NSE), curso e ano frequentado pelo estudante. Do total da amostra ( $\mathrm{n}=1240)$, sendo da universidade portuguesa $(n=533)$ e da universidade brasileira $(n=707)$, a maioria dos estudantes era do sexo feminino (54.9\%). A maior parcela dos alunos inquiridos enquadrava-se no grupo etário dos 19-20 anos (39.4\%), o mínimo foi de 17 anos e o máximo 56 anos, ou seja, estamos perante estudantes relativamente jovens que ambicionam adquirir conhecimentos e que procuram uma melhor qualidade de vida, ou condição financeira. Sobre o estado civil 94.1\% eram solteiros e $95.0 \%$ não tinham filhos. Salienta-se que $6.6 \%$ dos estudantes viviam sozinhos e quanto à coabitação dos alunos em tempo de aulas, verificamos que $47.5 \%$ residem com os amigos em tempo de aulas, numa casa/apartamento (46.3\%), mantidos pela família através de mesada (41.8\%), embora 41,2\% asseguravam a sua manutenção financeira através de bolsa de estudo ou atividade acadêmica. A maioria dos estudantes do presente estudo é pertencente à classe média alta (46.7\%). No que diz respeito à deslocação dos estudantes para a universidade, a maioria deslocava-se de transporte público (autocarro/ônibus) (37.1\%) e 36.5\% deslocavam-se a pé.

\section{Competências de estudo}

Relativamente à questão «Fora de sala de aula em média quantas horas faz. de estudo diário?», a grande maioria da amostra em estudo respondeu 1 hora de estudo (26,6\%), logo seguido pelo grupo de estudantes que assinala estudar 2h/dia (24,4\%). Na categoria de maior número de horas de estudo diário (Mais de 5h/ dia), apenas se enquadravam 2,9\% dos estudantes. Este cenário repete-se nas duas universidades, com o maior grupo de estudantes tanto na universidade brasileira (26,1\%), como na universidade portuguesa $(27,3 \%)$, a assinalar que em média estudam $1 \mathrm{H} /$ dia, sendo a proporção da categoria «Mais de $5 \mathrm{~h} /$ dia», de novo a que obteve menores percentuais, respetivamente, $2,4 \%$ e $3,5 \%$.

No estudo comparativo do tempo de estudo dos alunos entre as universidades dos dois países, o teste Mann-Whitney revelou diferenças estatísticas muito significativas $\mathrm{p} \leq 0,014$, sendo que a média de ordenação da universidade brasileira $(611,69)$ foi mais elevada do que a da universidade portuguesa $(573,70)$, significando que os estudantes brasileiros estudam mais tempo fora da sala de aula. Na comparação das proporções de estudantes das duas universidades enquadrados nas diferentes categorias de tempo de estudo (opções de resposta) também se observaram diferenças estatísticas muito significativas ( $\mathrm{x}^{2}: \mathrm{p} \leq$ 0,007), sendo que a proporção de estudantes da universidade brasileira que se enquadram na categoria de 3 horas é superior à da Universidade Portuguesa (21,2\% versus 13,4\%), ou seja os estudantes brasileiros estudam durante mais tempo. 
$\mathrm{Na}$ questão quantos livros leem por ano? a maior porcentagem dos alunos da Universidade Portuguesa são dos que leem 2 livros por ano (22\%), embora em segundo lugar a maior porcentagem é um dado alarmante, sendo dos alunos que não leêm nenhum livro por ano $(20,6)$ em relação aos alunos da universidade brasileira a maior porcentagem são dos que leêm de 4 a 6 livros por ano. No teste T-Studant apareceram diferenças altamente significativas $\mathrm{T}=\mathrm{p} \leq 0,000$, onde na universidade portuguesa a média de ordenação foi de $(3,77)$, e na universidade brasileira a média de ordenação foi de $(6,28)$ demonstrando uma leitura maior.

Quando questionados sobre Que tipo de livro lê?

No tipo de livro de fiç̧ão, nos testes $\mathrm{x}^{2} \mathrm{p} \geq 0,352$ não houve diferenças estatísticas significativas.

No tipo de livro de autoajuda, houve diferenças estatísticas altamente significativas $\mathrm{x}^{2} \mathrm{p} \leq 0.000$, onde na universidade brasileira aparece mais 9,4 casos que o esperado.

No tipo de livro de não ficção, houve também diferenças estatísticas altamente significativas $\mathrm{x}^{2} \mathrm{p} \leq$ 0.000 , onde na universidade brasileira aparecem mais 7,2 casos que o esperado.

Os alunos que assinalaram que não leem nenbum tipo de livro, houve também diferenças estatísticas altamente significativas $\mathrm{x}^{2} \mathrm{p} \leq 0.000$, onde na universidade portuguesa apareceram mais 6,65 casos que o esperado. Analisando a estatística descritiva o tipo de livro mais escolhido tanto na universidade portuguesa $(60,2 \%)$ e brasileira $(57,6 \%)$ foi o tipo de fiç̧ão.

Quanto às medidas de tendência central e de dispersão da pontuação do total da amostra (duas universidades) dos três fatores que constituem a ECE (Almeida \& Joly, 2013), a maior média foi obtida pelo F3, que também obteve uma moda e um valor máximo mais elevados, significando que os estudantes investem mais nos comportamentos e nas preocupações no sentido de viabilizarem um estudo competente e bem-sucedido. Quando analisamos estas medidas em cada uma das universidades em estudo, observamos que quer a média, a mediana e a moda dos três fatores são mais elevados na universidade Brasileira, o que quer dizer que estes estudantes têm mais competências de estudo. No entanto essas diferenças não são estatisticamente significativas.

Tabela I. Medidas de tendência central e de dispersão dos fatores da Escala de Competências de Estudo

\begin{tabular}{|c|c|c|c|c|c|}
\hline \multicolumn{6}{|c|}{ Universidade Brasileira } \\
\hline $\mathrm{Fl}$ & $22,07 \pm 7,350$ & 24,0 & 25,0 & 0 & 30 \\
\hline F2 & $21,80 \pm 7,243$ & 23,0 & 25,0 & 0 & 30 \\
\hline F3 & $27,97 \pm 8,460$ & 30,0 & 36,0 & 0 & 36 \\
\hline \multicolumn{6}{|c|}{ Universidade Portuguesa } \\
\hline $\mathrm{Fl}$ & $21,86 \pm 5,995$ & 23,0 & 20,0 & 0 & 30 \\
\hline F2 & $21,38 \pm 5,930$ & 22,0 & 20,0 & 0 & 30 \\
\hline F3 & $27,53 \pm 6,902$ & 29,0 & 30,0 & 0 & 36 \\
\hline \multicolumn{6}{|l|}{ Total da amostra } \\
\hline $\mathrm{Fl}$ & $21,98 \pm 6,799$ & 24,0 & 25,0 & 0 & 30 \\
\hline F2 & $21,62 \pm 6,711$ & 23,0 & 25,0 & 0 & 30 \\
\hline F3 & $27,78 \pm 7,829$ & 30,0 & 30,0 & 0 & 36 \\
\hline
\end{tabular}


No que diz respeito à comparação entre as duas universidades em termos das competências de estudo, constatamos que as médias do fator Comportamentos Estratégicos de Planejamento das duas universidades não diferem estatisticamente ( $\mathrm{t}$ de Student: $\mathrm{p} \geq$ ). O mesmo sucedeu no que se refere ás médias do fator Comportamentos Estratégicos de Monitoração das duas universidades ( $\mathrm{t}$ de Student: $\mathrm{p} \geq$ ). Repetiram-se os mesmos resultados relativamente ao fator Comportamentos Estratégicos de Autoavaliação (t de Student: $\mathrm{p} \geq$ ). Ou seja as competências de estudo dos estudantes Portugueses e dos estudantes Brasileiros não diferem significativamente, sendo bastante semelhantes entre si. Tais resultados estão na mesma direção dos estudos de Prates (2011) com sua amostra de 814 universitários, onde afirma que «leva à conclusão de que os universitários não apresentaram grande variabilidade em suas respostas». Estes resultados também corroboram com os estudos de Dias \& Joly (2013) que verificaram que sua amostra de 653 universitários que de uma forma geral, declararam utilizar de comportamentos estratégicos de estudo, tanto com relação ao planejamento, ao monitoramento e autoavaliação, porém ainda assim afirmaram que «aventa-se a hipótese de que os universitários mesmo relatando a utilização de comportamentos estratégicos de autorregulação, podem não estar sabendo aplicálas adequadamente quando encontram dificuldades em seu estudo ou problemas que precisam resolver para dar continuidade para atingir a aprendizagem de um conteúdo». (p.8).

Numa ótica geral entre as duas universidades e as 16 questões da escala, a questão mais pontuada (49,1\%) foi quando me aparece importante faço anotações nas aulas. E a questão com mais discordância (4,7\%) foi Procuro anotar aspectos que não compreendo para depois pedir ajuda ou voltar a eles e superar as dificuldades. Estes dados foram exatamente os mesmos nos estudos de Prates (2011), onde apresenta a questão menos pontuada e a mais pontuada.

Estudos realizados têm demonstrado a autorregulação como um aspecto decisivo para o desempenho melhor e consequente sucesso acadêmico dos estudantes universitários (Lindner \& Harris, 1993; Pintrich, Smith, Garcia \& Mckeachie, 1993). Porém, as pesquisas têm revelado que os estudantes ingressam na universidade com poucas competências para assumirem uma abordagem mais profunda na sua aprendizagem e para autorregular o seu estudo de forma eficaz (Almeida, Guisande, et al., 2009; Pintrich \& Zusho, 2002).

\section{Considerações Finais}

Tendo em conta os objetivos delineados para este estudo e analisando os resultados obtidos podemos retirar um conjunto de conclusões que passamos a descrever de seguida.

O perfil dos alunos que frequentam a universidade Portuguesa e que participaram neste estudo, pode considerar-se ser um estudante do sexo feminino, pertence ao grupo etário de 19 a 20 anos, coabita com amigos numa casa/apartamento mantido pela família, recebe mesada da família e pertence ao NSE médio alto, pelo que a universidade aparenta ser um espaço elitista. O perfil dos estudantes que frequentavam a Universidade Brasileira em estudo, pode considerar-se do sexo feminino, pertencente ao grupo etário de 18 e 19 anos, coabita com os colegas numa casa/apartamento mantido pela família, porém, sua manutenção financeira é proveniente de bolsa de estudos e/ou atividade académica e pertence ao NSE classe média.

Apesar dos estudantes Brasileiros despenderem em média mais tempo em estudo fora da sala de aula, e lerem mais livros do que os estudantes Portugueses, concluímos que em relação às competências de estudo, quanto ao planejamento, monitoramento e autoavaliação, não se constataram diferenças significativas entre os estudantes das duas universidades, pelo que os seus comportamentos, em termos de estratégias de estudo são muito semelhantes, no tipo de livro de leitura os alunos das duas universidades prefeririam mais a ficção. 
Os resultados obtidos poderão contribuir para que os docentes que lecionam, possam ficar a conhecer melhor os seus estudantes e adequar melhor as suas estratégias de ensino-aprendizagem, melhorando o processo de ensino aprendizagem, o sucesso académico dos estudantes, bem como o incentivo à leitura e estudo. Neste mundo global em que vivemos e com a proposta da internacionalização e mobilidade, este estudo oferece um recorte, uma breve visão das semelhanças e diferenças sociodemográficas e de competências de estudo entre dois países.

\section{Referências bibliográficas}

Almeida, Leandro (2002): "Facilitar a aprendizagem: ajudar os alunos a aprender e a pensar". Psicologia Escolar e educacional, 6 (2), 155-165.

Almeida, Leandro S. \& Soares, Ana Paula (2004): “Os estudantes universitários: Sucesso escolar e desenvolvimento psicossocial” in Mercuri, E \& Polydoro, S. (eds.): Estudante universitário: Características e experiências de formação. Taubaté: Cabral Editora e Livraria Universitária.

Almeida, Leandro et al.,(2009): "Escala de competências de estudo (ECE-SUP): fundamentos e construção". Actas do X Congresso Internacional Galego-Português de Psicopedagogia. Braga: CIEd Editora.

Almeida, Leandro S. et al., (2012): "Democratização do acesso e do sucesso no Ensino Superior: Uma reflexão a partir das realidades de Portugal e do Brasil”. Avaliação, 17 (3), 899-920.

Almeida, Leandro S. \& Joly, M. C. R. A. (2013): "Escala de competências de estudo - ECE (S\&H)". Teste em construção. Universidade do Minho (Portugal)/Universidade de Brasília (Brasil).

Carrasco, J. B. (1989). Como avaliar a aprendiragem. Coleção Práticas Pedagógicas. Porto: Edições Asa.

Credé, Marcus \& Kuncel, Nathan (2008): "Study Habits, Skills, and Attitudes: The Pillar Supporting Collegiate Academic Performance”. Perspectives on Psychological Science, 3 (6), 425-453.

Dias, Anelise Silva \& Joly, Maria Cristina Rodrigues Azevedo (2013): "Habilidades cognitivas para a competência de estudo”. Congresso Internacional Galego-Português de Psicopedagogia. Braga: CIEd Editora.

Ferreira, Joaquim Armando; Almeida, Leandro S. \& Soares, Ana Paula (2001): "Adaptação Acadêmica em estudante do $1^{\circ}$ ano: Diferenças de género, situação de estudante e curso". PsicoUSF, 6 (1), 01-10.

Ferreira, Carlos Alberto (2007). A avaliação no Quotidiano da sala de aula. Porto: Porto Editora.

Fortin, Marie Fabienne; Côté, José \& Filion, F. (2013). Fundamentos e etapas do processo de investigação. Loures: Lusodidacta.

Graffar, M. (1956): “Une methode de classification sociale d'échantillons de population”. Courrier, 6(8), 455-459.

Instituto Brasileiro De Geografia e Estatística (2015). Disponível em: http://www.ibge.gov.br/home, acesso em 20 de dezembro 2016.

Instituto Nacional de Estudos e Pesquisas Educacionais (2014). Censo da educação superior: Resumo técnico. Disponível em: http://download.inep.gov.br/download/superior/censo/2012/resumo_tecnico_censo_educacao_superior_2012.pdf, acesso em 18 dezembro 2016. 
Jenschke, B. (2003): “A cooperação internacional: Desafios e necessidades da orientação e do aconselhamento em face das mudanças mundiais no trabalho e na sociedade". Revista Brasileira de Orientação Profissional, 4, 35-55.

Lindner, Reinhard W. \& Harris, Bruce (1993): "Self-regulated learning: its assessment and instructional implications". Educational Research Quaterly, 16, 29-37.

Lopes, Carlos Alberto \& Pinto, Maria (2010): IL-HUMASS - Instrumento de avaliação de competências em literacia da informação: um estudo de adaptação à população portuguesa (Parte I) in $10^{\circ}$ Congresso de Bibliotecários, Arquivistas e Documentalistas, Guimarães. Lisboa: BAD. Disponível em http:/ / repositorio.ispa.pt/handle/10400.12/200, acesso em 3 janeiro 2017.

Macedo, A. R., et al., (2005): "Educação superior no século XXI e a reforma universitária brasileira". Ensaio: Avaliação de Políticas Públicas em Educação, 13 (47), 127-148.

Maestro, Valther (2014). Palestra trata do perfil universitário no século xxi. http://uaumais.com.br/palestra-trata-do-perfil-universitario-no-seculo-xxi, acesso em 8 novembro.

Marôco, João (2014). Análise estatística com o SPSS Statistics. Pêro Pinheiro: Report Number.

Méndez, F. (1999). Aprender a estudiar. Madrid: Ediciones Pirámide.

Ministério da Educação. (2007). O plano de desenvolvimento da educação: Razão, princípios e programa. Disponível em: http://portal.mec.gov.br/arquivos/pdf/livromiolov4.pdf, acesso em 5 Novembro 2016.

Nardelli, Giovanna. Gaudenci et al., (2013): "Perfil dos alunos ingressantes dos cursos da área da saúde de uma universidade federal". REAS, 2(1), 3-12.

Oliveira, J. B. (2006): Aprendizagem auto-regulada, envolvimento e ganhos académicos em estudantes do ensino superior. Tese de doutoramento não publicada, Universidade de Aveiro. https:/ ria.ua.pt/ bitstream/10773/4752/1/ 2007001149.pdf, acesso em 2 setembro 2016.

Pinheiro, Maria do Rosário Manteigas e Moura (2003). Uma época especial: Suporte social e vivências académicas na transição e adaptação ao ensino superior. Tese de doutoramento não publicada, Faculdade de Psicologia e Ciências de Educação, Universidade de Coimbra. http://hdl.handle.net/10316/988, acesso em 3 novembro 2016.

Pinto, M. (2010): "Design of the IL-HUMASS survey on information literacy in higher o education: a selfassessment approach". Journal of Information Science, 38 (1), 86-103.

Pinto, Carlos Lopes Maria (2011): "An approach to the internal facet of information literacy using the ILHUMASS survey: design of the IL-HUMASS survey". Journal of Academic Librarianship, 37 (2), 145-154.

Pinto, Carlos Lopes Maria (2012): "Information literacy perceptions and behaviour among history students”. Aslib Proceedings, 64 (3), 304-327.

Pintrich, Paul R. et al., (1993): "Reability and predictive validity of the motivated strategies for learning questionnaire (MSLQ)”. Educational and Psychological Measurement, 53, 801-813.

Pintrich, Paul R. (1999): “The role of motivation in promoting and sustaining self-regulated learning”. International Journal of Educational Research, 459-470. 
Pintrich, Paul R. \& Zusho, A. (2002): "A. The development of academic self-regulation: The role of cognitive and motivational factors" in Allan Wigfield \& Jacquelynne Eccles (eds.): Development of achievement motivation. San Diego: Academic Press.

Pintrich, P. R. (2004): "A conceptual framework for assessing motivation and selfregulated learning in college students". Educational Psychology Review, 16 (4), 385-407.

PORDATA Base de Dados Portugal contemporâneo. 2014. Disponível em: http:/ /www.pordata.pt/Subtema/Portugal/Alunos+Matriculados+do+Ensino+Superior-74, acesso em 4 janeiro 2017.

PORDATA Base de Dados Portugal contemporâneo. 2017. Disponível em: http:/ /www.pordata.pt/Subtema/Portugal/Alunos+Matriculados+do+Ensino+Superior-74. Acesso em 23 janeiro 2017.

Prates, Eli Andrade Rocha (2011): "Estudo de validade da escala de competência em estudo - ECE-SUP $(\mathrm{S} \& H)$ pela correlação com a motivação de universitários”. Tese de mestrado. Itatiba: Universidade São Francisco.

Robbins, S et al., (2004): "Do Psychosocial and study skill factors predict college outcomes? A metaanalysis". Psychological Bulletin, 130 (2), 261-288.

Rojas, Héctor Lamas (2008): “Aprendizaje autorregulado, motivación y rendimiento académico”. Liberabit. Revista de Psicología, 14, 15-20.

Santos, Luísa (2000). Vivencias académicas e rendimento escolar: Estudo com alunos universitários do $1^{\circ}$ ano. Dissertação de mestrado não publicada, Universidade do Minho, Braga. http://hdl.handle. net/1822/206, acesso em 20 Dezembro 2016.

Sarriera, Jorge Castellá, Paradiso, Ângela Carina, et al., (2012): "Estudo comparativo da integração ao contexto universitário entre estudantes de diferentes instituições”. Revista Brasileira de Orientação Profissional, $13(2), 163-172$.

Schreiber, F. J. (2005): "Metacognition and self-regulation in literacy" em S. E. Israel, C. C. Block, K. L. Bauserman \& K. Kinnucan-Welsch (orgs.): Metacognition in literacy learning: theory, assessment, instruction and professional development. Philadelphia: Lawrence Erlbaum.

Silva, Katia Regina Xavier \& Silva, Douglas Bordalo (2015): "Estratégias de autorregulação da aprendizagem no curso de educação física do UNIABEU Centro Universitário”. Gestão \& Sociedade: Revista de Pós-Graduação da Uniabeu, 4 (1), 56-71.

Soares, D. H. P. (2000): “As diferentes abordagens em orientação profissional” in M. D. Lisboa \& D. H. P. Soares (eds.): Orientação profissional em ação: Formação e prática de orientadores. São Paulo.

Soares. M. (2002). "Novas práticas de leitura e escrita: letramento na cibercultura". Educação \& Sociedade, $23(81), 143-160$.

UNESCO (2011). "Institute for Statistics. Global Education Digest: Comparing education statistics across the world". Quebec: Author. Disponível em: http://www.uis.unesco.org/Education/GED\%20Documents\%20C/GED-2011-Book-EN-web2.pdf, acesso em 3 agosto 2016. 
Veiga Simão, Ana Margarida (2004): “O conhecimento estratégico e a auto-regulação da aprendizagem. Implicações em contexto escolar” in Adelina Lopes da Silva, António Manuel Duarte, Isabel Antunes de Sá \& Ana M. Veiga Simão: Aprendizagem autorregulada pelo estudante: perspectivas psicológicas e educacionais. Porto: Porto Editora.

Zago, N. (2006): "Do acceso a permanência no ensino superior: percursos de estudantes universitários de camadas populares". Revista Brasileira de Educaşão, 11 (32), 226-237.

Zabalza, Miguel (2004). O ensino universitário: Seu cenário e seus protagonistas. Porto Alegre: Artmed.

Zimmerman, Barry (2000): "Attainment of self-regulation: a social cognitive perspective" in Monique Boekaerts, Moshe Zeidner, Paul R Pintrich: Handbook of self-regulation. San Diego: Academic Press.

Zimmerman, Barry \& Martinez-Pons, Manuel (1988). "Construct validation of a strategy model of student self-regulated learning”. Journal of Educational Psychology, 80, 284-290.

Zimmerman, Barry (2008). "Investigating self-regulation and motivation: historical background, methodological developments, and future propects". American Educational research Journal, 45 (1), 166-183.

\section{Notas biográficas}

Rubia Salheb Fonseca es doctoranda en Ciencias de la Educación en la Universidade de Trás-OsMontes e Alto Douro (UTAD), en Portugal. Máster en Ciencias de la Educación. Pedagoga y psicopedagoga. Miembro del grupo de Investigación del Centro de Investigación en Estudos da Criança (CIEC) en la Universidade do Minho.

Joaquim José Jacinto Escola es Doctor en Educación. Profesor en el Departamento de Educação e Psicologia de la Universidade de Trás-Os-Montes e Alto Douro (UTAD), en Portugal. Miembro del Grupo de Investigación reconocido en la Universidad de Salamanca «Educación Comparada y Políticas Educativas (ECPES)» y del gupo de investigación IF de la Universidade do Porto.

Amâncio Carvalho es Doctor en Estudios del Niño, con especialización en Salud Infantil. Profesor en la Escuela de Salud de la Universidade de Tras-os-Montes e Alto Douro (UTAD), en Portugal. Pertenece al Centro de Investigación en Estudios del Niño (CIEC).

Armando Loureiro es Doctor en Educación. Profesor en el Departamento de Educação e Psicologia la Universidade de Trás-Os-Montes e Alto Douro (UTAD), en Portugal. Pertenece al grupo de investigación KIDE- Conhecimento, Inovação e Diversidades em Educação de la Universidade do Porto. 